\title{
openheart Alcohol consumption and risk of incident heart failure in older men: a prospective cohort study
}

\author{
S Goya Wannamethee, ${ }^{1}$ Peter H Whincup,${ }^{2}$ Lucy Lennon, ${ }^{1}$ Olia Papacosta, ${ }^{1}$ \\ A Gerald Shaper ${ }^{1}$
}

To cite: Wannamethee SG, Whincup PH, Lennon L, et al. Alcohol consumption and risk of incident heart failure in older men: a prospective cohort study. Open Heart 2015;2: 000266 . doi:10.1136/openhrt-2015000266

Received 12 March 2015 Revised 1 July 2015 Accepted 7 July 2015

\section{CrossMark}

\footnotetext{
${ }^{1}$ Department of Primary Care and Population Health, University College London, London, UK

${ }^{2}$ Department of Population Health Sciences and Education, St George's, University of London, London, UK
}

Correspondence to Professor S Goya Wannamethee; g.wannamethee@ucl.ac.uk

\section{ABSTRACT}

Aims: Light-to-moderate drinking has been associated with reduced risk of heart failure (HF). We have examined the association between alcohol consumption and incident HF in older British men. Methods and results: Prospective study of 3530 men aged $60-79$ years with no diagnosed HF or myocardial infarction (MI) at baseline and followed up for a mean period of 11 years, in whom there were 198 incident HF cases. Men were divided into 6 categories of alcohol consumption: none, $<1,1-6,7-13,14-34$ and $\geq 35$ drinks/week. There was no evidence that light-to-moderate drinking is beneficial for risk of HF. Heavy drinking ( $\geq 35$ drinks/week) was associated with significantly increased risk of HF. Using the large group of men drinking 1-6 drinks/week as the reference group, the relative HRs (95\% confidence interval) for HF adjusted for age, lifestyle characteristics, blood pressure, atrial fibrillation and renal dysfunction were 0.97 (0.59 to 1.63), 1.39 (0.86 to 2.25), 1.00, 0.94 (0.64 to 1.43), 1.16 (0.78 to 1.71 ) and 1.91 (1.02 to $3.56)$ for the 6 alcohol groups, respectively. The increased risk associated with heavy drinking was attenuated after adjustment for $\mathrm{N}$-terminal pro-brain natriuretic peptide (NT-proBNP) (HR=1.43 (0.76 to 1.69)). Stratified analysis showed heavy drinking was associated with increased HF risk only in those with ECG evidence of myocardial ischaemia.

Conclusions: There was no evidence that light-tomoderate drinking is beneficial for the prevention of HF in older men without a history of an MI. Heavier drinking ( $\geq 5$ drinks/day), however, was associated with increased risk of $\mathrm{HF}$ in vulnerable men with underlying myocardial ischaemia.

\section{INTRODUCTION}

Heart failure (HF) is a major and increasingly important public health problem in older people and is associated with considerable hospitalisation and mortality. ${ }^{1}$ Although it is well documented that high levels of alcohol intake (generally $>90 \mathrm{~g} /$ day alcohol or more than 9 UK standard drinks) and alcohol abuse can result in alcoholic cardiomyopathy leading to $\mathrm{HF}^{2-4}$ a meta-analysis and several

\section{KEY MESSAGES}

What is already known about this subject?

- Light-to-moderate drinking is associated with reduced risk of heart failure (HF) in middle-aged populations. High levels of alcohol, usually more than $90 \mathrm{~g} /$ day (>9 standard UK drinks), and alcohol abuse can result in alcoholic cardiomyopathy leading to HF. Less is known about the influence of alcohol on $\mathrm{HF}$ in older adults in whom incident HF is high, and the level of alcohol intake which increases $\mathrm{HF}$ risk is uncertain.

What does this study add?

- We have examined the influence of light-tomoderate drinking and heavy drinking on risk of incident $\mathrm{HF}$ in an older population in whom HF is often not preceded by a myocardial infarction (MI), and assessed the effects of alcohol on $\mathrm{N}$-terminal pro-brain natriuretic peptide (NT-proBNP) (marker of cardiac dysfunction). The study does not provide evidence that light-to-moderate drinking is protective in older adults, and that drinking 5 or more drinks is associated with significantly increased risk of $\mathrm{HF}$ compared with lighter drinkers in those with underlying ischaemia.

How might this impact on clinical practice?

- Heavier drinking (5 or more drinks/day), particularly in those with underlying ischaemia, should be avoided. There is no evidence for a beneficial effect of light-to-moderate drinking to prevent $\mathrm{HF}$ in men aged over 60 years who have no history of an MI, although there was also no evidence of harm from light-to-moderate drinking in those without MI.

prospective studies, predominantly from the USA and mostly conducted in middle-aged populations, have suggested that light-tomoderate drinking may be beneficial for risk of $\mathrm{HF}^{5-12}$ However, this pattern was not observed in a large Finnish study ${ }^{13}$ and in the SAVE trial, ${ }^{14}$ and some studies suggest that no beneficial effect on incident HF is seen with light or moderate drinking (1-2 drinks/day) 
when HF is not preceded by a myocardial infarction (MI) ${ }^{11}$ This suggests that the lower risk of HF associated with light-to-moderate drinking may be mediated through beneficial effects of alcohol on coronary heart disease (CHD). ${ }^{11}$ However, few studies have examined the association between alcohol drinking and risk of HF in older adults, in whom the occurrence of HF with preserved ejection fraction is more common than HF with reduced ejection fraction ${ }^{15}$ and in whom HF is less likely to be associated with CHD. ${ }^{16}$ Moreover, in most population-based cohort studies which have examined the association between alcohol consumption and $\mathrm{HF}$ risk, heavier drinking ( $>3$ drinks/day) is underrepresented and the level of alcohol intake which is associated with increased HF risk is less certain although prospective population-based studies have suggested that the risk of developing atrial fibrillation, a major risk factor for HF, increases significantly with 5 or more drinks/ day ${ }^{17}$ and at an even lower threshold ( $>3$ drinks/day) in those with cardiovascular (CV) disease (CVD). ${ }^{18}$ We have, therefore, examined the association between alcohol consumption and incident $\mathrm{HF}$ in a prospective study of older British men aged 60-79 years with particular focus on whether heavy drinking is associated with increased HF risk compared with lighter drinking.

\section{PARTICIPANTS AND METHODS}

The British Regional Heart Study is a prospective study involving 7735 men aged 40-59 years drawn from one general practice in each of 24 British towns, who were screened between 1978 and $1980 .{ }^{19}$ The population studied was socioeconomically representative of British men and comprises predominantly white Europeans $(>99 \%)$. In 1998-2000, all surviving men, now aged 60-79 years, were invited for a 20th year follow-up examination. Ethical approval was obtained from all relevant local research ethics committees. All men completed a mailed questionnaire providing information on their lifestyle and medical history, had a physical examination and provided a fasting blood sample. The samples were frozen and stored at $-20^{\circ} \mathrm{C}$ on the day of collection and transferred in batches for storage at $-70^{\circ} \mathrm{C}$ until analysis, which was carried out after no more than one freezethaw cycle. The 12-lead ECGs were recorded using a Siemens Sicard 460 instrument and were analysed using Minnesota Coding definitions at the University of Glasgow ECG core laboratory. ${ }^{20}$ The men were asked whether a doctor had ever told them that they had angina, MI (heart attack, coronary thrombosis), HF or stroke; details of their medications were recorded at the examination. In total, 4252 men (77\% of survivors) presented for examination. Blood measurements were available for 4045 men. We excluded 117 men with prevalent HF and a further 395 men with a history of doctordiagnosed MI, and 3 men with no information on alcohol intake. We excluded men with prevalent MI because these men are at particularly high risk of $\mathrm{HF}$ and are particularly likely to receive advice to change their lifestyle, including alcohol intake. The analyses are thus based on 3530 men.

\section{CV risk factors at 1998-2000}

Anthropometric measurements, including body weight and height, were carried out with participants standing in light clothing without shoes. Details of measurement and classification methods for smoking status, physical activity, social class, blood pressure, blood lipids and forced expiratory volume in $1 \mathrm{~s}\left(\mathrm{FEV}_{1}\right)$ in this cohort have been described..$^{21-23}$ Prevalent diabetes included men with a diagnosis of diabetes and men with fasting blood glucose $\geq 7 \mathrm{mmol} / \mathrm{L}$. Predicted glomerular filtration rate (eGFR) was estimated from serum creatinine $;{ }^{24}$ GFR $=186 *(($ creatinine $) * *-1.154) *(($ age $) * *$ $-0.203)$. N-terminal pro-brain natriuretic peptide (NT-proBNP) was determined using the Elecsys 2010 electrochemiluminescence method (Roche Diagnostics, Burgess Hill, UK) ${ }^{23}$ NT-proBNP measurements were performed at baseline, and not in close relation to incident events. Electrocardiographic left ventricular hypertrophy (LVH) was defined according to relevant Minnesota Codes (codes 3.1 or 3.3). Atrial fibrillation was defined according to Minnesota Codes 8.3.1 and 8.3.3. Evidence of myocardial ischaemia on ECG was based on Minnesota Codings 1.1-1.3 (definite, probable or possible MI) or 4.1-4.4 and 5.1-5.3 (definite, probable or possible myocardial ischaemia). In total, 102 men had silent MI on ECG with no history of a doctor diagnosis of MI; these men were included in the study.

\section{Alcohol intake}

The men were asked to describe their current frequency of drinking (daily, most days, weekend only, occasional, special occasions only or none) and were asked to estimate the number of alcoholic drinks during an average week. The men were classified into six groups according to their reported weekly intake: none $(\mathrm{n}=344),<1 \quad(\mathrm{n}=338), 1-6$ $(\mathrm{n}=1259), 7-13(\mathrm{n}=712), 14-34(\mathrm{n}=740)$ and $\geq 35$ drinks/ week $(\mathrm{n}=137)$. One UK drink $=10 \mathrm{~g}$ alcohol. Light-to-moderate drinking refers to those drinking up to 34 drinks/week. Heavy drinking is defined as drinking $\geq 35$ drinks/week ( 5 or more drinks daily). To achieve sufficient numbers and because previous report suggest that risk of atrial fibrillation, a major risk factor for HF, is only elevated at levels of 5 drinks or more a day, we have used $\geq 35$ drinks as the threshold for heavy drinking. We have used the large group of 1-6 drinks/week as the reference group for comparison purposes. The alcohol questionnaire used in this study has been validated using 25 biochemical and haematological measurements on a single blood sample which indicated that the reported levels of alcohol consumption were valid on a group basis. ${ }^{25}$

\section{Follow-up}

All men have been followed up from initial examination (1978-1980) for CV morbidity and development of 
diabetes and follow-up has been achieved for $99 \%$ of the cohort. ${ }^{26}$ In the present analyses, all-cause mortality and morbidity events are based on follow-up from the rescreening examination in 1998-2000 to July 2010, a mean follow-up period of 11 years (range 10-12 years). Information on death was collected through the established 'tagging' procedures provided by the National Health Service registers. Fatal CHD events were defined as death with CHD (International Classification of Diseases (ICD), Ninth Revision, codes 410-414) as the underlying code. A non-fatal MI was diagnosed according to WHO criteria. ${ }^{27}$ Evidence of non-fatal MI and HF was obtained by ad hoc reports from general practitioners supplemented by biennial reviews of the patients' practice records (including hospital and clinic correspondence) through to the end of the study period. Possible or probable cases were not included in incident CHD cases. Incident non-fatal HF was based on a confirmed doctor diagnosis of HF from primary care records and where possible, verified using details of available clinical information from primary and secondary care records (including symptoms, signs, investigations, treatment response) to ensure that the diagnosis was consistent with current recommendations on $\mathrm{HF}$ diagnosis. ${ }^{28}$ These data were available for 160 of the 194 non-fatal cases $(82 \%)$. The incidence and determinants of HF cases identified using this process has already been reported and are consistent with results from other studies. ${ }^{22}{ }^{23}$ Incident HF included incident non-fatal HF (194 cases) as well as death from HF (4 cases) as the underlying cause (ICD, Ninth revision, code 428).

\section{Statistical methods}

The $\chi^{2}$ tests and the analysis of variance were used to assess the difference in baseline characteristics between the six alcohol groups. Cox's proportional hazards model was used to assess the multivariate-adjusted HR (relative risk) by alcohol categories. The large group of men drinking 1-6 drinks/week was used as the reference group. The proportional hazards assumption was examined using time-varying covariates, calculating interactions of predictor variables and a function of survival time and including these in the models. Examination of time-varying covariates indicated no violation of the proportionality assumption in the sample. In multivariate analyses, smoking (never-smokers, long-term ex-smokers ( $>15$ years), recent ex-smokers ( $<15$ years) and current smokers), social class (manual vs non-manual), physical activity (4 groups), diabetes (yes/no), use of antihypertensive treatment (yes/no), prior stroke (yes/no), diagnosed angina (yes/no), LVH (yes/no), renal dysfunction (yes/no), and atrial fibrillation (yes/no) were fitted as categorical variables. Systolic blood pressure, body mass index, $\mathrm{FEV}_{1}$ and NT-proBNP were fitted continuously. To evaluate whether alcohol predicted $\mathrm{HF}$ independent of incident MI (ie, those who developed MI during follow-up), we adjusted for incident non-fatal MI, fitting this as a time-dependent covariate.

\section{RESULTS}

During the mean follow-up period of 11 years, there were 198 incident $\mathrm{HF}$ cases (rate 5.9/1000 person-years) and 336 major CHD events in the 3530 men with no doctor diagnosis of HF or MI at baseline (ie, prevalent MI).

Table 1 shows the baseline characteristics by levels of alcohol consumption. Overall non-drinkers and heavy drinkers ( $\geq 35$ drinks/week) tended to have the most adverse CV risk factors. In particular the non-drinkers were the oldest group and had the highest prevalence rates of physical inactivity, manual workers, stroke, atrial fibrillation and renal dysfunction. They also had the highest mean levels of NT-proBNP. Since NT-proBNP is strongly associated with age $(\mathrm{r}=0.40 ; \mathrm{p}<0.0001)$, adjustment for age attenuated the increased NT-proBNP levels in non-drinkers and increased the mean levels in heavy drinkers, who then showed the highest NT-proBNP levels. Men drinking $<1 \mathrm{drink} /$ week in general had characteristics close to those of non-drinkers. There was an inverse association between alcohol intake and the percentage of men who developed CHD during follow-up.

Figure 1 shows the Kaplan-Meier curve of the cumulative incidence of $\mathrm{HF}$ by levels of alcohol consumption in men without a doctor diagnosis of MI. Heavy drinkers showed the highest cumulative incidence of $\mathrm{HF}$ followed by those drinking none or $<1$ drink/week. Incidence rates and relative $\mathrm{HR}$ for $\mathrm{HF}$ at different levels of alcohol consumption, using the large group of men consuming (1-6 drinks/week) as the reference group, are shown in table 2. In age-adjusted analyses, nondrinkers and infrequent drinkers showed higher risk, albeit non-significantly, compared with those drinking 1-6 drinks/week. Adjustment for CV risk factors (model 3) attenuated the increased HF risk in non-drinkers and these showed similar risk to both groups of light drinkers (1-6 and 7-13 drinks/week groups). Heavy drinkers showed significantly increased HF risk, but this persisted even after adjustment for these CV risk factors. Further adjustments for $\mathrm{FEV}_{1}$ (model 4) and in particular NT-proBNP (model 5) attenuated the increased relative risk of $\mathrm{HF}$ associated with heavy drinking. Further adjustment for incident MI strengthened the association in heavy drinkers, but it remained non-significant. Exclusion of all men who had ever reported heavy drinking ( $>6$ drinks/day since entry to the study at 40 59 years) in model 2 made little difference to the findings. We carried out further analysis separating the 2134 drinks/week from the 15-34 drinks/week category. The adjusted relative risk (95\% confidence interval) were 1.00 (0.60 to 1.67$), 1.43$ (0.88 to 2.31 ), $1.00,1.02$ (0.67 to 1.57$), 1.48$ (0.94 to 2.34$), 0.57$ (0.25 to 1.31 ) and 1.80 (0.96 to 3.36) for the seven groups: none, $<1$, $1-6, \quad 7-14, \quad 15-20, \quad 21-34$ and $\geq 35$ drinks/week, respectively.

We also examined the association between alcohol and HF stratified by age ( $<70$ vs $\geq 70$ years) using non-drinkers and men drinking $<1$ drink/week combined as the 
Table 1 Baseline characteristics by alcohol consumption in men without prevalent diagnosed MI or HF

\begin{tabular}{|c|c|c|c|c|c|c|c|}
\hline & \multirow[b]{2}{*}{$\begin{array}{l}\text { Non-drinker } \\
(\mathrm{N}=344)\end{array}$} & \multirow[b]{2}{*}{$\begin{array}{l}<1 \\
(\mathrm{~N}=338)\end{array}$} & \multicolumn{3}{|c|}{ Current alcohol intake (drinks/week) } & \multirow[b]{2}{*}{$\begin{array}{l}\geq 35 \\
(N=137)\end{array}$} & \multirow[b]{2}{*}{ p difference } \\
\hline & & & $\begin{array}{l}1-6 \\
(N=1259)\end{array}$ & $\begin{array}{l}7-14 \\
(N=712)\end{array}$ & $\begin{array}{l}15-34 \\
(N=740)\end{array}$ & & \\
\hline Age (years) & $69.7(5.5)$ & $68.8(5.3)$ & $68.6(5.5)$ & $68.5(5.5)$ & $67.8(5.2)$ & $67.4(5.2)$ & $<0.0001$ \\
\hline BMI $\left(\mathrm{kg} / \mathrm{m}^{2}\right)$ & $26.8(4.3)$ & $26.7(3.7)$ & $26.6(3.4)$ & $26.8(3.4)$ & $27.1(3.6)$ & $27.0(3.8)$ & 0.20 \\
\hline Obese (\%) & 15.4 & 15.0 & 14.5 & 16.7 & 17.0 & 20.4 & 0.41 \\
\hline Current smokers (\%) & 18.7 & 16.3 & 10.0 & 11.4 & 12.9 & 22.2 & $<0.0001$ \\
\hline Inactive (\%) & 45.8 & 39.4 & 32.1 & 27.0 & 31.0 & 34.1 & $<0.0001$ \\
\hline Manual workers (\%) & 68.0 & 64.6 & 52.1 & 50.8 & 43.2 & 56.2 & $<0.0001$ \\
\hline Past >6 drinks/day drinkers (\%) & 3.2 & 2.0 & 3.7 & 11.6 & 23.9 & 66.4 & $<0.0001$ \\
\hline Stroke $(\%)$ & 8.1 & 2.7 & 4.2 & 4.4 & 5.6 & 2.9 & 0.008 \\
\hline Atrial fibrillation (\%) & 3.8 & 3.8 & 2.8 & 3.3 & 3.6 & 1.5 & 0.64 \\
\hline Angina (\%) & 11.6 & 10.0 & 8.3 & 7.3 & 8.7 & 3.7 & 0.05 \\
\hline Diabetes (\%) & 14.5 & 14.8 & 10.7 & 12.2 & 12.7 & 15.3 & 0.24 \\
\hline On BP- lowering treatment (\%) & 29.9 & 31.6 & 28.8 & 26.9 & 27.8 & 26.3 & 0.70 \\
\hline LVH (\%) & 9.0 & 6.2 & 8.0 & 7.4 & 6.8 & 9.5 & 0.60 \\
\hline ECG evidence of ischaemia (\%) & 27.1 & 20.8 & 21.9 & 22.0 & 21.9 & 25.3 & 0.08 \\
\hline $\mathrm{FEV}_{1}(\mathrm{~L})$ & $2.47(0.6)$ & $2.58(0.7)$ & $2.67(0.7)$ & $2.61(0.6)$ & $2.64(0.6)$ & $2.53(0.6)$ & $<0.0001$ \\
\hline Heart rate (bpm) & $67.1(12.5)$ & $67.0(13.4)$ & $64.6(12.2)$ & $65.4(12.4)$ & $66.6(13.2)$ & $69.1(13.6)$ & $<0.0001$ \\
\hline $\mathrm{SBP}(\mathrm{mm} \mathrm{Hg})$ & $148.4(22.7)$ & $147.3(23.2)$ & $149.4(24.7)$ & $150.0(24.0)$ & $152.5(22.9)$ & $157.4(22.9)$ & $<0.0001$ \\
\hline HDL-C (mmol/L) & $1.25(0.3)$ & $1.25(0.3)$ & $1.28(0.3)$ & $1.36(0.3)$ & $1.42(0.4)$ & $1.61(0.4)$ & $<0.0001$ \\
\hline GGT (IU/L)* & $25.8(18-33)$ & $25.2(17-34)$ & $26.3(18-35)$ & $29.1(19-37)$ & $33.8(25-46)$ & $45.6(27-31)$ & $<0.0001$ \\
\hline eGFR $\left(\mathrm{mL} / \mathrm{min} / 1.73 \mathrm{~m}^{2}\right)$ & $71.1(13.9)$ & $71.9(14.5)$ & $71.7(12.3)$ & $72.7(12.6)$ & $73.9(11.4)$ & $75.0(12.2)$ & $<0.0001$ \\
\hline Renal dysfunction (\%) & 20.4 & 15.5 & 15.7 & 12.7 & 10.8 & 13.1 & 0.0004 \\
\hline NT-proBNP $(p g / m L)^{*}$ & $105.6(45-207)$ & $88.2(41-183)$ & $85.6(41-165)$ & $87.4(45-157)$ & $86.5(42-159)$ & $101.5(51-198)$ & 0.04 \\
\hline (Age-adjusted) & $(95.6)$ & $(87.4)$ & $(85.6)$ & $(85.6)$ & $(89.1)$ & $(111.0)$ & 0.07 \\
\hline Developed CHD (\%) & 13.1 & 11.5 & 9.6 & 8.0 & 8.5 & 8.0 & 0.08 \\
\hline
\end{tabular}

Mean (SD) unless specified.

Data on alcohol not available in three men.

*Geometric mean and IQR.

BMl, body mass index; BP, blood pressure; CHD, coronary heart disease; eGFR, estimated glomerular filtration rate; FEV

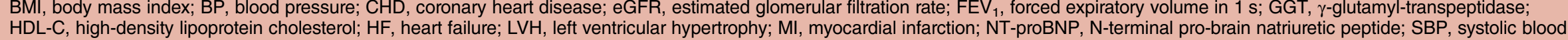
pressure. 
Figure 1 Kaplan-Meier curve of cumulative heart failure (HF) incidence by alcohol intake in men with no diagnosed myocardial infarction (MI). Log rank test $\mathrm{p}=0.14$. Drinks/week $1=$ none; $2=<1 ; 3=1-6 ; 4=7-14$; $5=15-34 ; 6=\geq 35$ (heavy). men with no pre-existing diagnosed $\mathrm{MI}$

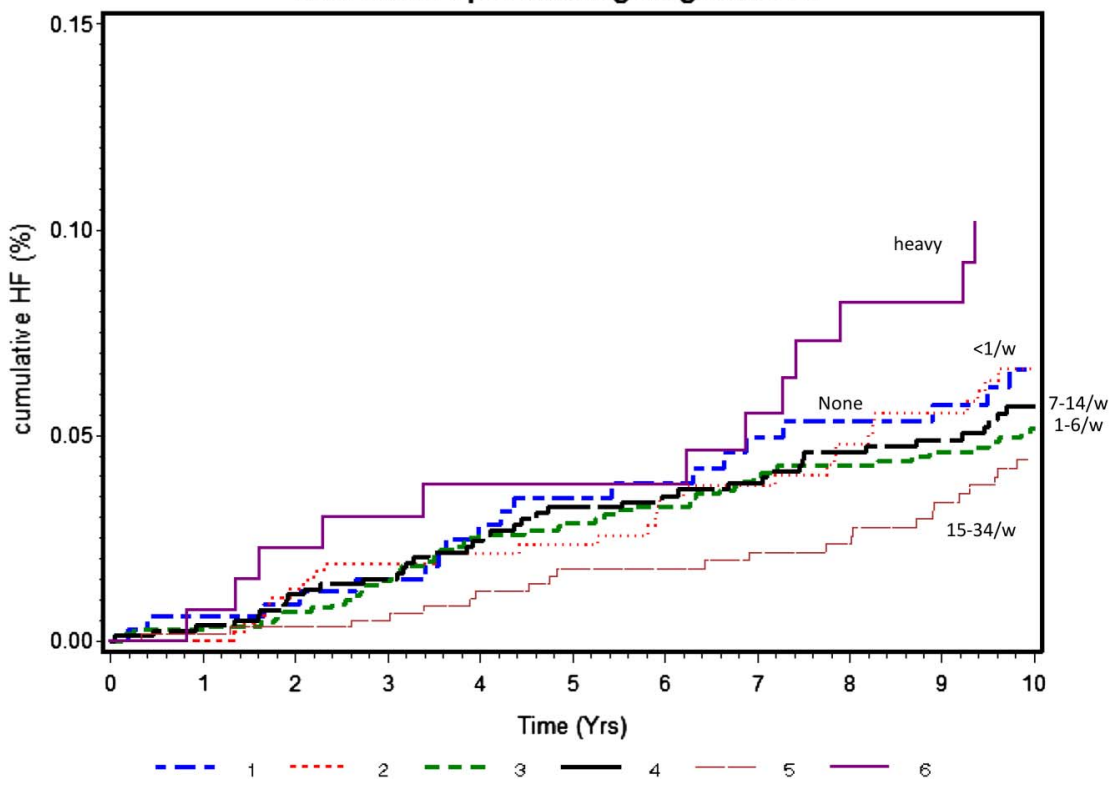

those $\geq 70$ years were $1.00,0.84$ ( 0.51 to 1.37$), 0.91(0.52$ to 1.61$)$ and $0.79(0.42$ to 1.48$)$ for none $/<1,1-6,7-14$ and 15-34 drinks/week groups, respectively.

Since NT-proBNP is a marker of cardiac damage, we further examined the association between alcohol intake and HF risk separately in men with and without ECG evidence of myocardial ischaemia (table 3). To achieve sufficient numbers, the non-drinkers and those reporting $<1 \mathrm{drink} /$ week were combined, and the 7-14 and 14-34 drinks/week groups were combined, creating four

Table 2 Heart failure rates/1000 person-years and adjusted HR for heart failure according to alcohol consumption in men with no pre-existing diagnosed myocardial infarction (MI) or heart failure

\begin{tabular}{|c|c|c|c|c|c|c|}
\hline & \multirow[b]{2}{*}{$\begin{array}{l}\text { Non-drinker } \\
(\mathrm{N}=344)\end{array}$} & \multicolumn{3}{|c|}{ Alcohol intake (drinks/week) } & \multirow[b]{2}{*}{$\begin{array}{l}15-34 \\
(N=740)\end{array}$} & \multirow[b]{2}{*}{$\begin{array}{l}\geq 35 \\
(N=137)\end{array}$} \\
\hline & & $\begin{array}{l}<1 \\
(\mathrm{~N}=338)\end{array}$ & $\begin{array}{l}1-6 \\
(\mathrm{~N}=1259) \\
\end{array}$ & $\begin{array}{l}7-14 \\
(\mathrm{~N}=712)\end{array}$ & & \\
\hline $\begin{array}{l}\text { Rates/1000 } \\
\text { person-years } \\
\text { (n) }\end{array}$ & $6.9(21)$ & $7.9(25)$ & $5.2(64)$ & $5.0(34)$ & $5.9(42)$ & $9.4(12)$ \\
\hline Age-adjusted & 1.19 (0.71 to 1.92$)$ & $1.54(0.96$ to 2.48$)$ & 1.00 & 0.98 (0.65 to 1.49$)$ & $1.17(0.80$ to 1.73$)$ & $2.04(1.10$ to 3.77$)$ \\
\hline Model 1 & 0.98 (0.59 to 1.63$)$ & $1.43(0.89$ to 2.32$)$ & 1.00 & 0.94 (0.62 to 1.43$)$ & $1.15(0.78$ to 1.70$)$ & $1.90(1.02$ to 3.54$)$ \\
\hline Model 2 & $1.01(0.61$ to 1.61$)$ & 1.49 (0.92 to 2.32$)$ & 1.00 & 0.95 (0.63 to 1.45$)$ & $1.19(0.81$ to 1.76$)$ & $1.89(1.01$ to 3.52$)$ \\
\hline Model 3 & 0.97 (0.59 to 1.63$)$ & 1.39 (0.86 to 2.25$)$ & 1.00 & 0.94 (0.64 to 1.43$)$ & $1.16(0.78$ to 1.71$)$ & $1.91(1.02$ to 3.56$)$ \\
\hline Model 4 & 0.94 (0.57 to 1.58$)$ & 1.44 (0.86 to 2.29$)$ & 1.00 & 0.93 (0.58 to 1.36$)$ & $1.14(0.76$ to 1.67$)$ & $1.80(0.96$ to 3.36$)$ \\
\hline Model 5 & $0.92(0.55$ to 1.56$)$ & $1.28(0.75$ to 2.10$)$ & 1.00 & 0.93 (0.61 to 1.43$)$ & $1.14(0.76$ to 1.69$)$ & $1.43(0.76$ to 1.69$)$ \\
\hline Model 6 & 0.93 (0.55 to 1.57$)$ & 1.38 (0.84 to 2.29$)$ & 1.00 & $1.02(0.66$ to 1.56$)$ & 1.22 (0.82 to 2.82$)$ & $1.56(0.81$ to 3.00$)$ \\
\hline $\begin{array}{l}\text { Model } 2 \text { and } \\
\text { exclusion of } \\
\text { past heavy } \\
\text { drinkers (>6 } \\
\text { drinks/day) }\end{array}$ & 0.95 (0.57 to 1.58$)$ & $1.38(0.85$ to 2.24$)$ & 1.00 & $0.99(0.65$ to 1.51$)$ & $1.15(0.76$ to 1.74$)$ & 1.83 (0.98 to 3.40$)$ \\
\hline \multicolumn{7}{|c|}{$\begin{array}{l}\text { Model 1: adjusted for age, smoking, body mass index, social class, prevalent stroke, diabetes and angina. } \\
\text { Model 2: Model 1+left ventricular hypertrophy, antihypertensive drugs and systolic blood pressure. } \\
\text { Model 3: Model 2+atrial fibrillation and renal dysfunction. } \\
\text { Model 4: Model 3+forced expiratory volume in } 1 \mathrm{~s} \text {. } \\
\text { Model 5: Model 4+N-terminal pro-brain natriuretic peptide (NT-proBNP). } \\
\text { Model 6: Model 5+incident MI. }\end{array}$} \\
\hline
\end{tabular}


Table 3 Age-adjusted mean NT-proBNP and adjusted HR for heart failure according to alcohol consumption in men with and without ECG evidence of ischaemia

\begin{tabular}{|c|c|c|c|c|}
\hline & \multicolumn{3}{|l|}{ Alcohol intake } & \multirow[b]{2}{*}{$\geq 35 /$ week } \\
\hline & $<1-$ week & 1-6/week & 7-34/week & \\
\hline & \multicolumn{4}{|c|}{ Men without ECG evidence of myocardial ischaemia } \\
\hline $\mathrm{N}$ (cases) & $519(25)$ & $983(43)$ & $1142(48)$ & $102(4)$ \\
\hline Age-adjusted mean NT-proBNP & 78.3 & 73.7 & 75.2 & 88.2 \\
\hline Model 1 & 0.94 (0.56 to 1.57$)$ & 1.00 & $1.01(0.67$ to 1.53$)$ & $0.99(0.55$ to 2.79$)$ \\
\hline Model 2 & 0.84 (0.49 to 1.43$)$ & 1.00 & $1.02(0.67$ to 1.54$)$ & $0.83(0.29$ to 2.36$)$ \\
\hline \multirow[t]{2}{*}{ Model 2 (exclude past heavy drinkers) } & $0.92(0.52$ to 1.64$)$ & 1.00 & $0.97(0.63$ to 1.51$)$ & $0.78(0.27$ to 2.21$)$ \\
\hline & \multicolumn{4}{|c|}{ Men with ECG evidence of myocardial ischaemia } \\
\hline $\mathrm{N}$ (cases) & $163(21)$ & $276(21)$ & $310(28)$ & $35(8)$ \\
\hline Age-adjusted mean NT-proBNP & 148.4 & 145.4 & 148.0 & 219.2 \\
\hline Model 1 & 1.60 (0.85 to 3.02$)$ & 1.00 & $1.11(0.62$ to 2.00$)$ & $3.53(1.52$ to 8.24$)$ \\
\hline Model 2 & $1.62(0.84$ to 3.11$)$ & 1.00 & $1.13(0.62$ to 2.03$)$ & 3.11 (1.27 to 7.57$)$ \\
\hline Model 2 (excluding silent MI) & $1.43(0.69$ to 2.95$)$ & 1.00 & $1.02(0.54$ to 1.90$)$ & 3.01 (1.15 to 7.87$)$ \\
\hline Model 2 (exclude past heavy drinkers) & 1.85 (0.88 to 3.88$)$ & 1.00 & $1.32(0.68$ to 2.54$)$ & $3.40(1.36$ to 8.50$)$ \\
\hline \multicolumn{5}{|c|}{$\begin{array}{l}\text { Model 1: adjusted for age, smoking, BMI, social class, prevalent stroke, diabetes, angina, LVH, antihypertensive drugs, systolic blood } \\
\text { pressure, atrial fibrillation, renal dysfunction and FEV }{ }_{1} \text {. } \\
\text { Model 2: Model } 1+N T-p r o B N P . \\
\text { Past heavy drinkers=those ever reporting drinking } \geq 6 \text { drinks/day. } \\
\text { BMl, body mass index; FEV }{ }_{1} \text {, forced expiratory volume in } 1 \mathrm{~s} ; \mathrm{LVH} \text {, left ventricular hypertrophy; MI, myocardial infarction; NT-proBNP, } \\
\text { N-terminal pro-brain natriuretic peptide. }\end{array}$} \\
\hline
\end{tabular}

alcohol groups overall. Evidence of myocardial ischaemia was present in about $22 \%$ of the men with no diagnosed MI. Heavy drinking was associated with increased risk of HF only in those with ECG evidence of myocardial ischaemia and this remained even after adjustment for NT-proBNP and exclusion of men with silent MI $(n=102)$. No association was seen at all between alcohol intake and HF risk in those with no evidence of myocardial ischaemia. Exclusion of heavy drinkers made little difference to the findings in men with no myocardial ischaemia. In those with ischaemia, those drinking $<1$ drink/week showed higher risk compared with those drinking 1-6 drinks/week, but the difference was not significant. We carried out a sensitivity analysis restricting the analyses to the 160 validated cases. This did not materially change the results. The adjusted relative risks (model 1) for the four alcohol groups in those with no ischaemia were 1.02 (0.59 to 1.78$), 1.00,1.14$ (0.72 to $1.78)$ and 1.22 (0.43 to 3.48) respectively, and $1.61(0.80$ to 3.23 ), $1.00,1.19$ (0.63 to 2.24 ) and 3.54 (1.36 to 9.23 ) in those with ischaemia, respectively.

\section{DISCUSSION}

In this study of older British men, there was no evidence that light-to-moderate drinking (up to 4 drinks/day) was associated with a beneficial effect on $\mathrm{HF}$ risk overall; non-drinkers showed similar risk to light/moderate drinkers (up to 34 drinks/week). Heavy drinking ( $\geq 35$ drinks/week or $\geq 5$ drinks/day), however, was associated with a significantly increased risk of HF compared with those drinking 1-6 drinks/week, which was largely seen in those with ECG evidence of myocardial ischaemia. Our findings extends those of previous studies by examining the effects of both light-to-moderate drinking as well as heavy drinking on risk of $\mathrm{HF}$ and the role of NT-proBNP, a marker of cardiac damage, and underlying myocardial ischaemia.

\section{Light-to-moderate drinking and HF}

Previous studies mainly from the USA have suggested that light-to-moderate drinking is associated with a beneficial effect on $\mathrm{HF}^{5-12}$ However, recent findings from a large Finnish cohort showed no beneficial effect of light drinking on HF similar to the current findings. ${ }^{13}$ Most of these previous studies were conducted in middle-aged populations. In one study it was suggested that the benefit of light-to-moderate drinking is due to its effect on reducing $\mathrm{CHD}$, which in turn is associated with reduced rates of $\mathrm{HF}$ rather than a direct beneficial effect of alcohol on HF risk. ${ }^{11}$ In the US Kaiser Permanente study, light-to-moderate drinking was associated with reduced risk of $\mathrm{HF}$ associated with coronary artery disease (CAD), but no benefit was seen for light-to-moderate drinking and the risk of non-CADrelated $\mathrm{HF}$, and heavy drinking $>3$ drinks/day increased risk of non-CAD-related $\mathrm{HF}^{7}$ Older patients with $\mathrm{HF}$ differ from younger patients in that a higher proportion of older patients with $\mathrm{HF}$ have $\mathrm{HF}$ with preserved ejection fraction. ${ }^{15}$ These patients are less likely to have CHD and more likely to have hypertension and atrial fibrillation. ${ }^{16}$ A high proportion of men without a history of a doctor diagnosis of an MI in this study who developed HF did not develop an MI before developing HF $(85 \%)$, which would explain the difference in findings between this and the younger US cohorts. In contrast, CHD is the predominant risk factor for HF with reduced ejection fraction, which is more common in 
younger adults. ${ }^{16}$ This may explain the overall lower risk of $\mathrm{HF}$ associated with light-to-moderate drinking seen in many of the previous studies which have been conducted in younger populations. The association between alcohol and HF in older adults has been less well studied. In the Cardiovascular Health Study of men aged $>65$ years, a weak but non-significant inverse association was seen between light-to-moderate alcohol intake and HF in those without CVD. ${ }^{8}$

The magnitude of the health effects of alcohol is dependent on the base group used; non-drinkers and infrequent drinkers are usually not an appropriate group to estimate the effects of alcohol. ${ }^{29}$ For comparison purposes with other studies, when non-drinkers and infrequent drinkers were used as the comparison group, we showed a weak and non-significant protective effect of light-to-moderate drinking on HF risk in this older population. Our findings are not dissimilar to that seen in the Cardiovascular Health Study. However, the inconsistent findings that non-drinkers and the infrequent drinkers had different risk patterns for HF does not provide convincing evidence for a protective effect of alcohol on $\mathrm{HF}$ in older men. This is in contrast to CHD events where there was a clear inverse relation between alcohol drinking and the percentage of men who developed an MI in this study, as expected. Moreover, there was no association at all between alcohol intake and HF in the large group of men with no evidence of myocardial ischaemia. While we cannot exclude the possibility that light-tomoderate drinking may have some protective effect in men with ischaemia, we cannot confirm this in our study.

\section{Heavy drinking, NT-proBNP and HF risk}

It is well established that excessive alcohol intake, usually defined as $>90 \mathrm{~g} /$ day (more than 9 standard UK drinks), can lead to cardiomyopathy, ${ }^{3}$ which in turn leads to left ventricular dysfunction and $\mathrm{HF}^{2}$ Most studies on heavy drinking and cardiomyopathy have been conducted in alcoholic patients, in whom detectable changes in cardiac structure are seen in those who report drinking $>90 \mathrm{~g} /$ day. $^{3}$ However, few studies have been able to examine the impact of heavier drinking below this threshold on HF risk in the general population because of the low prevalence of heavy drinkers in these study populations. Most population studies in the US which have investigated the association between alcohol and incident HF have focused on the benefits of light drinking and no increased risk is seen with heavier drinking, usually defined as just $>2$ drinks/day. However, in the US Kaiser Permanente study, heavy drinking defined as $>3$ drinks/day was shown to be associated with non-CAD-related $\mathrm{HF}^{7}$ In a Swedish population study, men who reported history of alcohol abuse showed increased risk of $\mathrm{HF}^{30}$ We have shown that risk of $\mathrm{HF}$ is increased in men consuming 5 or more drinks/day, the majority of whom reported drinking $<8$ drinks/day. These findings are in keeping with those from the Copenhagen Study which showed that risk of incident atrial fibrillation, a major risk factor for HF, is only increased at levels of 5 drinks or more. ${ }^{17}$ We observed no association between alcohol intake and prevalent atrial fibrillation. However, this may be due to the fact that many men with atrial fibrillation are likely to develop stroke or CVD, and have reduced their intake as a result of developing CVD.

NT-proBNP, a peptide released from myocardium in response to ventricular wall stress and dysfunction, is a marker of myocardial damage and subclinical cardiac function, and is strongly related to incident atrial fibrillation and $\mathrm{HF}^{23}{ }^{31}$ A recent report has shown NT-proBNP to be increased in heavy drinkers in a population-based study $^{32}$ which is consistent with our findings. We have shown that the effect of heavy drinking on incident HF risk was to a large extent associated with NT-proBNP. This may reflect a direct toxic effect of alcohol on the myocardium with consequent effects on cardiac function and ventricular wall stress, as has been suggested. ${ }^{32}$ Further subsidiary analysis showed that heavy drinking ( $\geq 5$ drinks/day) increased risk only in those with ECG evidence of myocardial ischaemia and this was only partly due to raised NT-proBNP. Thus, regular heavy drinking appears to have adverse effects on HF risk, particularly in the presence of myocardial ischaemia where it may aggravate the myocardial damage or dysfunction leading to HF.

\section{Limitations}

There are several limitations in this study. The current findings are based on doctor-diagnosed HF, which is likely to underestimate the true incidence of HF in this study population; however, these diagnoses are usually supported by evidence from hospital admissions and hospital attendances investigation. These men who were survivors of an ongoing cohort study and attended the re-examination may have been healthier than the general older population which might have also affected the absolute incident rates of HF. However, this should not have affected the associations between alcohol and HF risk; our previous reports on HF predictors, such as obesity and NT-proBNP, in this cohort have generally accorded with prior data and therefore, suggest external validity for our findings. ${ }^{22} 23$ Echocardiographic measurements were not carried out in the present study and we were not able to differentiate $\mathrm{HF}$ with reduced ejection fraction and $\mathrm{HF}$ with preserved ejection fraction. We did not have information on incident atrial fibrillation to assess the possible mediating role of atrial fibrillation. Although we did not see a significant effect for light-to-moderate drinkers, the number of men drinking $<1$ week in this study was small and the CIs do not exclude such an effect completely because of the size and power of the study. Although we excluded past heavy drinkers, higher levels of alcohol consumption may have been under-reported, leading to a misclassification of heavier drinkers into lower consumption categories. Such a misclassification may have attenuated any 
protective effects present among true light-to-moderate drinkers. Finally, it was based on an older, predominantly white, male population of European origin, so that the results cannot be generalised directly to women, or to younger populations or other ethnic groups.

\section{CONCLUSION}

In this study of older British men, there is no evidence that light-to-moderate drinking is beneficial for the prevention of $\mathrm{HF}$ in men without $\mathrm{MI}$; heavier drinking $\geq 5$ drinks/day is associated with increased risk of $\mathrm{HF}$, particularly in men with underlying myocardial ischaemia.

Contributors SGW initiated the concept and design of the study, and drafted the manuscript. AGS contributed to the concept of the study and drafting of manuscript. OP contributed to the statistical analysis of the data. LL and PHW contributed to the acquisition of the study data and drafting of the manuscript.

Funding The British Regional Heart Study is a British Heart Foundation (BHF) research group and receives support from BHF Programme grant RG/08/013/ 25942.

\section{Competing interests None declared.}

Patient consent Obtained.

Ethics approval Local research ethics committees.

Provenance and peer review Not commissioned; externally peer reviewed.

Data sharing statement Data request is considered by the British Regional Heart Study committee team. Contact I.lennon@ucl.ac.uk.

Open Access This is an Open Access article distributed in accordance with the terms of the Creative Commons Attribution (CC BY 4.0) license, which permits others to distribute, remix, adapt and build upon this work, for commercial use, provided the original work is properly cited. See: http:// creativecommons.org/licenses/by/4.0/

\section{REFERENCES}

1. McMurray JJ, Stewart S. Epidemiology, aetiology and prognosis of heart failure. Heart 2000;83:596-602.

2. George A, Figueredo VM. Alcoholic cardiomyopathy: a review. J Card Fail 2011;17:844-9.

3. Lacovoni A, de Maria R, Gavazzi A. Alcoholic cardiomyopathy. J Cardiovasc Med (Hagerstown) 2010;11:884-92.

4. Laonigro I, Correale M, di Biase M, et al. Alcohol abuse and heart failure. Eur J Heart Fail 2009;11:453-62.

5. Abramson JL, Williams SA, Krumholz HM, et al. Moderate alcohol consumption and risk of heart failure among older persons. JAMA 2001;285:1971-7.

6. Walsh CR, Larson MG, Evans JC, et al. Alcohol consumption and risk for congestive heart failure in the Framingham Heart Study. Ann Intern Med 2002;136:181-91.

7. Klatsky AL, Chartier D, Udaltsova N, et al. Alcohol drinking and risk of hospitalization for heart failure with and without associated coronary artery disease. Am J Cardiol 2005;96:346-51.

8. Bryson CL, Mukamal KJ, Mittleman MA, et al. The association of alcohol consumption and incident heart failure: the Cardiovascular Health Study. J Am Coll Cardiol 2006;48:305-11.

9. Djousse L, Gaziano JM. Alcohol consumption and heart failure in hypertensive US male physicians. Am J Cardiol 2008;102:593-7.

10. Gonçalves A, Claggett B, Jhund PS, et al. Alcohol consumption and risk of heart failure: the Atherosclerosis Risk in Communities Study. Eur Heart J 2015;36:939-45.

11. Djousse L, Gaziano JM. Alcohol consumption and risk of heart failure in the physician's Health Study 1. Circulation 2007;115:34-9.
12. Larsson SC, Orsini N, Wolk A. Alcohol consumption and risk of heart failure: a dose-response meta-analysis of prospective studies. Eur J Heart Fail 2015;17:367-73.

13. Wang $Y$, Tuomilehto J, Jousilahti $P$, et al. Lifestyle factors in relation to heart failure among Finnish Men and women. Circ Heart Fail 2011;4:607-12.

14. Aguilar D, Skali H, Moyé LA, et al. Alcohol consumption and prognosis in patients with left ventricular systolic dysfunction after a myocardial infarction. J Am Coll Cardiol 2004;43:2015-21.

15. Kaila K, Haykowsky MJ, Thompson RB, et al. Heart failure with preserved ejection fraction in the elderly: scope of the problem. Heart Fail Rev 2012;17:555-62.

16. Brouwers FP, de Boer RA, van der Harst $P$, et al. Incidence and epidemiology of new onset heart failure with preserved vs. reduced ejection fraction in a community-based cohort: 11-year follow-up of PREVEND. Eur Heart J 2013;34:1424-31.

17. Mukamal KJ, Tolstrup JS, Friberg J, et al. Alcohol consumption and risk of atrial fibrillation in men and women: the Copenhagen City Heart Study. Circulation 2005;112:1736-42.

18. Liang Y, Mente A, Yusuf S, et al. for the ONTARGET and TRANSCEND Investigators. Alcohol consumption and the risk of incident atrial fibrillation among people with cardiovascular disease. CMAJ 2012;184:E857-66.

19. Shaper AG, Pocock SJ, Walker M, et al. British Regional Heart Study: cardiovascular risk factors in middle-aged men in 24 towns. Br Med J (Clin Res Ed) 1981:283:179-86.

20. Macfarlane PW, Devine B, Latif S, et al. Methodology of ECG interpretation in the Glasgow program. Methods Inf Med 1990;29:354-61.

21. Wannamethee SG, Lowe GDO, Whincup PH, et al. Physical activity and hemostatic and inflammatory variables in elderly men. Circulation 2002;105:1785-90.

22. Wannamethee SG, Shaper AG, Whincup PH, et al. Obesity and risk of incident heart failure in older men with and without pre-existing coronary heart disease: does leptin have a role? J Am Coll Cardiol 2011;58:1870-7.

23. Wannamethee SG, Welsh $\mathrm{P}$, Whincup $\mathrm{P}$, et al. N-terminal pro brain natriuretic peptide but not copeptin improves prediction of heart failure over other routine clinical risk parameters in older men with and without cardiovascular disease: population-based study. Eur $J$ Heart Fail 2014;16:25-32.

24. Levy AS, Bosch JP, Lewis JB, et al. A more accurate method to estimate glomerular filtration rate from serum creatinine: a new prediction equation. Modification of Diet in Renal Disease Study Group. Ann Intern Med 1999:130:461-70.

25. Shaper AG, Pocock SJ, Ashby D, et al. Biochemical and haematological response to alcohol intake. Ann Clin Biochem 1985;22:50-61.

26. Walker M, Shaper AG, Lennon L, et al. Twenty year follow-up of a cohort study based in general practices in 24 British towns. J Public Health Med 2000;22:479-85.

27. Rose G, Blackburn H, Gillum RF, et al. Cardiovascular survey methods. 2nd edn Geneva, Switzerland: World Health Organisation, 1982.

28. McMurray JJ, Adamopoulos S, Anker SD, et al. ESC Committee for Practice Guidelines. ESC Guidelines for the diagnosis and treatment of acute and chronic heart failure 2012: the Task Force for the Diagnosis and Treatment of Acute and Chronic Heart Failure 2012 of the European Society of Cardiology. Developed in collaboration with the Heart Failure Association (HFA) of the ESC. Eur Heart $J$ 2012;33:1787-847.

29. Fillmore KM, Stockwell T, Chikritzhs T, et al. Moderate alcohol use and reduced mortality risk: systematic error in prospective studies and new hypotheses. Ann Epidemiol 2007:17(5 Suppl):S16-23.

30. Wilhelmsen L, Rosengren A, Eriksson $\mathrm{H}$, et al. Heart failure in the general population of men-morbidity, risk factors and prognosis. J Intern Med 2001;249:253-61.

31. Patton KK, Heckbert SR, Alonso A, et al. N-terminal pro-B-type natriuretic peptide as a predictor of incident atrial fibrillation in the Multi-Ethnic Study of Atherosclerosis: the effects of age, sex and ethnicity. Heart 2013;99:1832-6.

32. Leon DA, Shkolnikov VM, Borinskaya S, et al. Hazardous alcoho consumption is associated with increased levels of B-type natriuretic peptide: evidence from two population-based studies. Eur $J$ Epidemiol 2013;28:393-404. 